\title{
Use of Navigation Beacons to Support Lunar Vehicle Operations
}

\author{
Evan Anzalone \\ NASA/MSFC \\ MSFC/EV42 \\ Huntsville, AL 35812 \\ evan.j.anzalone@nasa.gov
}

\author{
Anand Iyer \\ NASA/MSFC \\ MSFC/EV42 \\ Huntsville, AL 35812 \\ anand.r.iyer@nasa.gov
}

\author{
Tamara Statham \\ NASA/MSFC \\ MSFC/EV42 \\ Huntsville, AL 35812 \\ tamara.l.statham@nasa.gov
}

\begin{abstract}
To support a wide variety of lunar missions in a condensed regime, solutions are needed outside of the use of Earth-based orbit determination. This research presents an alternate approach to in-situ navigation through the use of beacons, similar to that used on Earth as well as under technology development efforts. An overview of the current state of navigation aids included as well as discussion of the Lunar Node - 1 payload being built at NASA/Marshall Space Flight Center. Expected navigation results of this beacon payload for planned operation from the lunar surface are provided. Applications of navigation beacons to multiple stages of the proposed human lunar landing architecture are given, with initial analysis showing performance gains from the use of this technology. This work provides a starting point for continued analysis and design, laying out the foundation of how navigation beacons can be incorporated into the architecture to enable continued analysis, design, and future expanded capability.
\end{abstract}

\section{TABLE OF CONTENTS}

1. INTRODUCTION. . .1

2. TERRESTRIAL BEACON APPROACHES .................... 1

3. SPACE APPLICATIONS ........................................ 4

4. LUNAR NODE - 1 ....................................................6 6

5. APPLICATIONS TO FUTURE MISSIONS ................... 7

6. NETWORK AND CAPABILITY EXPANSION ............. 10

7. SUMMARY ........................................................................ 11

ACKNOWLEDGEMENTS ................................................. 11

REFERENCES.......................................................... 11

\section{INTRODUCTION}

As human space exploration pushes back toward the moon and onward to Mars, the architectures for extended operations are continuing to mature and move towards implementation. As part of these missions, including human landers and ascent vehicles, an increased infrastructure will need to be deployed to support long duration scenarios. One aspect of this is the ability to communicate and navigate across the lunar surface. To enable this, a Lunar-wide communication network will be deployed, sizing up with the amount of bandwidth required for operations. This network can also be used for navigation as well. This paper provides analysis and description of a beacon network that can be integrated across spacecraft, fixed land sites, and mobile assets in order to spread these functions. A particular example and focus of this work is the development of the Lunar Node - 1 payload. This hardware implements a method of using communication networks to also provide navigation via the Multi-spacecraft Autonomous Positioning System. A description of the payload and its operations is provided as a summary. The larger focus of this paper is the application of this technology to a breadth of mission scenarios, including ground navigation, ascent vehicles, and precision landing.

This paper presents the application of beacons both in an orbital environment and fixed to the lunar surface for support of these mission scenarios. Traditional radiometric approaches will be used to provide a baseline comparison for the orbital application scenario. Using analysis simulations tools, potential vehicle trajectories are assessed and the additional capabilities of using beacons are described, including effects on sensor selection and integration. Potential cargo and human mission trajectories are assessed to capture expected performance. Lastly, the paper describes how the system can be used in various operational modes to support surface navigation, taking advantage of wellunderstood terrestrial approaches to improve state knowledge and provides a comparison to other potential methods. This work presents an architecture starting from a single landed beacon that can continue to support a broad range of missions and operational scenarios, growing into a distributed lunar navigation network.

\section{TERRESTRIAL BEACON APPROACHES}

\section{Navigation Approaches with Beacons}

Loran-C (the third iteration of LORAN, short for long-range radio navigation) is based on technology developed in the years after World War II as a ground-based radio system used by the military until it was largely superseded by satellitebased navigation, which had become far cheaper and more precise. It involves the use of at least three beacons at known locations that transmit a pulsed, low-frequency radio signal on the order of $100 \mathrm{kHz}$ [1], detected by a receiver on an aircraft. Unlike radar, the time it takes for the signal to reach the receiver from the ground station cannot be calculated. However, the time difference between pulse acquisitions for each station can be determined since the signals are synchronized. With one pair of stations, the aircraft is known 
to be on a point on a hyperbola, by definition [2]. With a third station (or a second pair of stations), one can pinpoint the exact location of the aircraft in 2D space. One advantage of Loran-C technology in a lunar environment is that the lack of atmosphere does not result in propagation errors and delays which require compensating adjustments on Earth. Furthermore, due to the accuracy of Loran-C over very long ranges, a lunar application is advantageous for crewed exploration and navigation.

A very high frequency (VHF) omnidirectional range radio (VOR) is another ground-based navigational aid that operates at much shorter ranges than Loran-C, due to its much higher operational frequency from 108-118 MHz [3], and it remains to this day the most commonly used navigation system in aviation. A VOR station sends out two signals simultaneously: an omnidirectional reference wave and a phased variable wave. While the former is frequency modulated (and is used to tune the receiver in to the correct VOR frequency), the latter is amplitude modulated, where the phase angle between the phased and reference signals determines the receiver's bearing to or from the station. A schematic of this system can be seen in Figure 1 below.

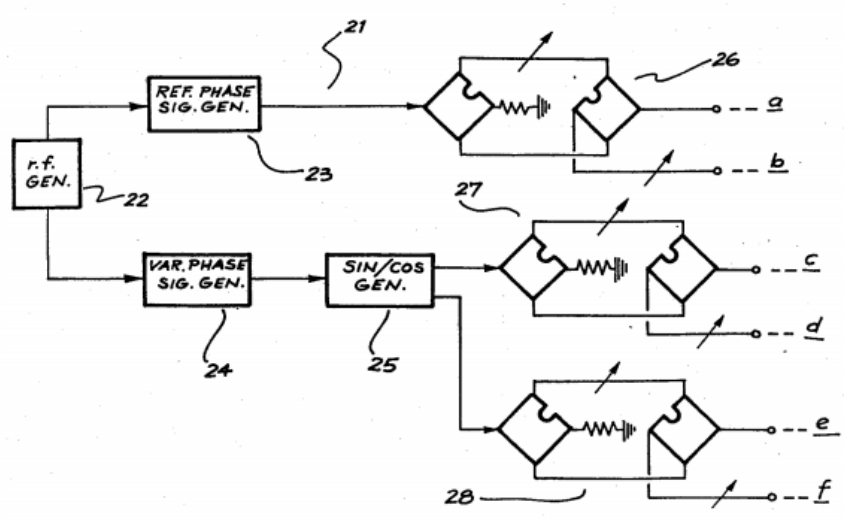

Figure 1 Patent Schematic of Complete VOR System [3]

Using any two VORs that are within range allows an aircraft to triangulate its position, while centering a course deviation indicator (CDI) located on the instrument panel allows the aircraft to laterally find and track a radial off of any single station. The angular nature of tracking a VOR radial means that the accuracy becomes more sensitive in close proximity to the station, producing large swings and fluctuations on the CDI when passing directly overhead, a phenomenon referred to by pilots as the "cone of confusion." VORs are often used in conjunction with distance measuring equipment (DME) or tactical air navigation systems (TACAN). With VOR/DME, an aircraft can fix its position in 2D space using a radial and a slant-range distance (which requires only one VOR) rather than the intersection point of two radials (which requires two VORs). A VOR/TACAN system (or VORTAC) is a much more precise military variant of a VOR/DME, and since they are also cleared for use in civil aviation, VORTACs are far more common than VOR/DME and VOR standalone systems. The downside to both DME and TACAN is that it uses slant-range, so an aircraft flying at 6000 feet directly overhead will receive the same DME measurement as an aircraft a mile out at 2850 feet. In other words, lateral accuracy decreases with altitude as well as with proximity to the station. VOR/DME systems with both vertical and lateral capabilities are implemented widely on instrument landing systems (ILS) and microwave landing systems (MLS) for shooting precision approaches in landing aircraft. Thus, using a VOR variant on the lunar surface is not only expedient for surface navigation, but can also serve as an aid in guiding precision landings on lunar descent vehicles.

Radio-direction finding (RDF) equipment is similar to Loran$\mathrm{C}$ in its extensive use of low frequency radio waves. Unlike a VOR, however, an RDF system only transmits a single signal, with no information regarding directionality, giving it the more common label of non-directional beacon (NDB). An antenna mounted exterior to the aircraft is first tuned to the desired frequency. The pilot (or operator for marine applications) then manually rotates the antenna until the weakest signal strength is determined, in a process known in aviation as null positioning, which will indicate the bearing to or from the station. NDBs transmit a vertically polarized electromagnetic wave, meaning that the electric field oscillates vertically while the magnetic field oscillates horizontally. The latter induces a voltage in the horizontallyoriented antenna as a function of phase angle, and the null position - and therefore, the beacon bearing - is established when the antenna has effectively aligned itself to the magnetic field. However, since an NDB, by nature, does not provide directional info, a second NDB is required to verify whether the established radial is to the station or from it. Then, similar to a VOR beacon sans DME, the 2D position can be triangulated and the pilot or operator can home in to the beacon instead of away from it. An automatic direction finder $(\mathrm{ADF})$ is a system that removes the need for the second NDB by incorporating a second antenna used solely for directional guidance once the NDB antenna has determined a radial. This ADF antenna is vertically oriented and is thus aligned with the electric field broadcasted by the beacon. It compares the NDB antenna signal with the voltage induced in itself by the electric field, and uses that information to resolve the beacon direction [4]. While ADF reduces the need for a second beacon for homing purposes, multiple beacons are still required to establish a fix due to the lack of a DME equivalent. In recent decades, ADF/NDB systems have been largely supplanted by more accurate and reliable VOR/DME and GPS units. However, like Loran-C, RDF and its derivatives are extremely practical for long-range operations, especially when dealing in situations outside of visual lineof-sight limits, including crewed scouting and sortie missions on other planets along with autonomous robotic operations.

While Loran-C, VOR, and RDF systems are all ground-based navigation, satellite-based navigation, primarily global positioning system (GPS), has become relatively inexpensive, exceptionally precise, and incredibly popular in recent years. Also a form of radio-based navigation, GPS provides the receiver with position in $3 \mathrm{D}$ space, along with 
time information. Initially a military application (and still owned and operated by the US Air Force), a civilian system requires line-of-sight to at least four satellites in order to obtain an accurate fix: the first satellite locates the receiver at some point on a sphere, the second satellite narrows it down to a circle, the third refines it to two points, and the fourth determines the correct point. All the satellites transmit a coded pseudorange to the receiver with clock errors built in, which effectively synchronizes the satellites and allows the receiver to filter out the propagation errors [5]. The basic pseudorange value is calculated as shown in Eq. 1 below:

$$
\rho_{i}=\sqrt{\left(x_{i}-x\right)^{2}+\left(y_{i}-y\right)^{2}+\left(z_{i}-z\right)^{2}}+c \Delta T+n_{i}
$$

where $\rho_{i}{ }^{*}$ is the measured pseudorange for satellite $i ; x_{i}, y_{i}$, and $\mathrm{z}_{\mathrm{i}}$ are the measured positions in a given Cartesian frame for satellite $\mathrm{i} ; \mathrm{x}, \mathrm{y}$, and $\mathrm{z}$ are the actual positions of the satellite in the same Cartesian frame; $c$ is the speed of light; $\Delta \mathrm{T}$ is the time differential between broadcast and reception; and $n_{i}$ is the noise for satellite $\mathrm{i}$.

However, the pseudorange transmissions do not take into account GPS signal integrity, so most aircraft have receiver autonomous integrity monitoring (RAIM), an aircraft-based augmentation system (ABAS) that uses several redundant GPS fixes and cross-checks them to evaluate anomalies [6]. Thus, RAIM generally requires at least five satellites (or four satellites and a barometric altimeter, known as baro-aiding) to isolate an incorrect signal. If a bad signal is detected, a sixth satellite (or a fifth with baro-aiding) is required to maintain RAIM after the bad satellite signal is excluded, a process known as fault exclusion. An alternative to ABAS is the satellite-based augmentation system (SBAS), such as wide-area augmentation system (WAAS), operated by the US Federal Aviation Administration (FAA). WAAS checks for GPS satellite errors on a ground master station at a known location. These errors are transmitted to communication satellites that rebroadcast the errors to the GPS receiver, which takes these errors into account when it receives position signals from GPS satellites. The integrity information provided by WAAS is far higher than that provided by RAIM, and is thus used by aircraft for precision approaches into airports. However, RAIM is more robust and fault-tolerant, so aircraft are required by the FAA to first perform a RAIM check before conducting any GPS approach (precision or otherwise) [7].

\section{Beacons for Inter-asset Awareness}

Automatic dependent surveillance-broadcast (ADS-B) is an aircraft flight following technology that uses satellites to determine and broadcast state information every second. The information that is broadcasted is referred to as "ADS-B Out," while information that is received is described as "ADS-B In." It is designed to serve as a backup to primary surveillance radar (PSR) and replace secondary surveillance radar (SSR) in busier airspaces. PSR is a standard groundbased pulse radar used by air traffic control (ATC) for aircraft surveillance by tracking aircraft position and bearing that updates only every 13 seconds, while SSR consists of data broadcasted from the aircraft itself (generally a Mode $\mathrm{C}$ altitude-encoding transponder). While PSR would still permit ground facilities to maintain watch in case of aircraft equipment failure, replacing SSR with ADS-B would impart ATC with augmented aircraft information, ensuring increased levels of safety in aviation. In fact, the FAA has mandated all civilian aircraft to be equipped with ADS-B Out in order to legally fly in most controlled airspace starting January 1, 2020. SSR is still required on aircraft as backup in case of an ADS-B malfunction [8], but the sheer amount of situational awareness provided by ADS-B is irrefutable. Some of the information it provides includes call sign (or tail number), latitude, longitude, geometric (or geodetic) altitude, barometric pressure altitude, horizontal velocity, vertical velocity, aircraft length, aircraft width, groundspeed, ground track, heading, instrument flight rules (IFR) capability, ADSB In capability, information integrity, timestamp, among other details [9]. Some approved variants of ADS-B include automatic dependent surveillance-rebroadcast (ADS-R), which relays ADS-B information between aircraft equipped with ADS-B In that are on different frequency links; traffic information services-broadcast (TIS-B), which is a trafficonly system that allows pilots to see positions and groundtrack of nearby aircraft; and flight information servicesbroadcast (FIS-B), which provides en-route and destination weather information and relevant meteorological data. ADSB not only amplifies situational awareness, but also facilitates ATC with aircraft operations. Basic traffic spacing, surface operations, clearances, and frequency congestion are all designed to become more efficient and streamlined with the addition of this technology, and it will also help to reduce runway incursions, airspace incursions, mid-air and ground collisions, and search-and-rescue response times, making civilian aviation an all-around safer domain, as displayed in Figure 2 below; taking a similar approach to lunar-based operations would be a prudent choice. While traffic and clearance problems may be less routine on the Moon, an ADS-B-derived system would greatly increase inter-asset awareness in an efficient manner both among crew members and between crew and autonomous robotic equipment.

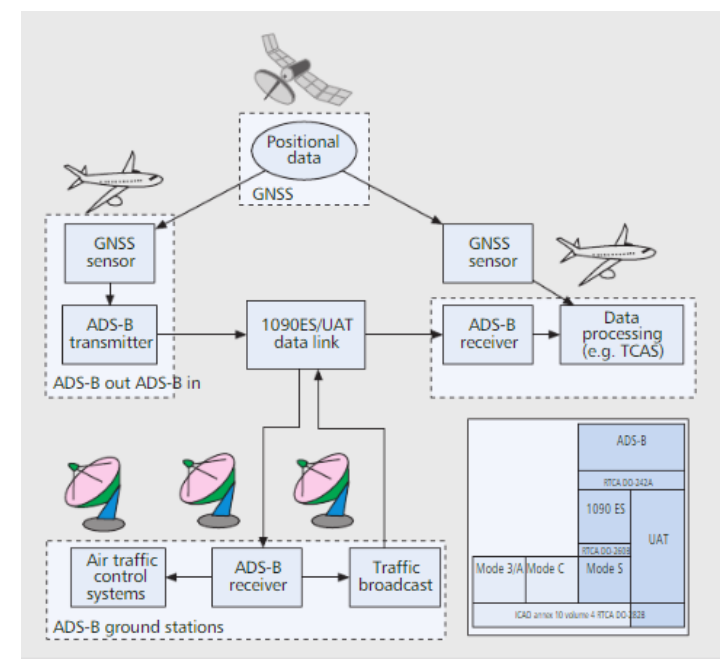

Figure 2 System Architecture and Protocol Hierarchy of 


\section{ATC with the Incorporation of ADS-B [8]}

A military analog of the ADS-B platform is the Joint Tactical Information Distribution System (JTIDS), which allows for an integrated communication, navigation, and identification network. It is the radio transmission and reception portion of a larger framework known as Tactical Digital Information Link J (TADIL J) [10]. JTIDS operates as a decentralized, time-division multiple-access (TDMA) system, allowing several aircraft and ground terminals to transmit nearly simultaneously on a common frequency channel using, as the name implies, separately assigned timeslots for their respective transmissions. Users all operate from a common network time reference (NTR), which is generally synchronized to Greenwich Mean Time (or, as it's referred to in the military and in aviation, "Zulu" time). Furthermore, JTIDS implements a spread-spectrum approach, a technique that artificially increases the bandwidth of a transmitted signal for a given frequency in order to overcome issues such as interference (noise) and signal detection and jamming. All of this is accomplished over a secure network called Link-16. Similar to other high-frequency systems like VOR and GPS, Link-16 has LOS limitations, but the key advantage is its versatility. It can not only transmit two-way voice data, but it can also serve as a method of transmitting text-based digital information without the necessity of separate, dedicated communication links [11]. JTIDS also includes relative navigation (relnav) capabilities for each terminal in the system. This enhances situational awareness for each aircraft by sharing all information about distances and relative bearings with each other. However, due to errors inherent to these measurements, high accuracy information is not necessarily guaranteed. Proposals have been made to increase its precision by integrating it with GPS as well as with round trip timing (RTT), a two-way measurement procedure between the transmitter and receiver that can be used to more accurately assess clock errors [12]. If successfully implemented on the moon, a variant of the robust JTIDS system combined with a lunar GPS or ADS-B spinoff would be incredibly strategic in providing a single system used for conditions requiring precision navigation simultaneously with communication and elevated situational awareness, such as for hazard avoidance maneuvers during terminal descent phases and for in-space rendezvous and docking operations.

\section{SPACE APPLICATIONS}

One of the most significant applications of spacecraft that builds on beacon tech is the Electra transceiver built as part of a local network capability for the Martian environment. Known as the Electra Proximity Link Payload when it flew on the Mars Reconnaissance Orbiter in 2005, the Electra is baselined at the ultra high frequency (UHF) range and is used for telecommunication relay between spacecraft as well as for radiometric navigation during mission-critical events, such as during the initial approach to the planet upon entering its sphere of influence as well as during entry, descent, and landing (EDL) phases. One of the features of Electra is its efficiency. A single unit weighs less than $5 \mathrm{~kg}$ and, in the worst case, draws 70 Watts, while still being able to receive signals at $-125 \mathrm{dBm}$ and delivering over $9 \mathrm{~W}$ across the band during transmissions [13]. For navigational determination, Electra uses precise two-way Doppler measurements to accurately determine relative in-space position and velocity down to within 300 meters or less [14], enabling capabilities for operations like precision landing and enhancing the probability of mission success. Not only is Electra compact and economical, but it has a platform based on softwaredefined radio (SDR), which gives the relay system a substantial amount of flexibility while mitigating the changes of hardware failure. This brings about another benefit, in that its integration with orbiting spacecraft allows for far higher data rates than conventional, increasing information transfer and minimizing lead time. The versatility of the Electra payloads combined with their stable operational parameters makes this system an excellent candidate to serve as a mobile counterpart to ground-fixed beacon networks.

Substantial work has also been done in the area of pseudosatellites (pseudolites), which are non-satellite systems that behave and perform the roles of a satellite, most commonly in the form of a ground-based transceiver. The challenge with implementing pseudolite systems on another planet like the Moon or Mars is calibrating each unit, for which the expected standard is centimeter-level accuracy. A feasible fix comes in the form of a self-calibrating pseudolite array (SCPA), an autonomous system wherein several pseudolite receivers communicate with and exchange GPS signals to determine their own 3D positions relative to each other down to the desired accuracy, and maintain it with minimal drift. Efforts led by Stanford University in this arena in the early 2000s have led to promising conclusions, although some challenges remain unaddressed. Prototype pseudolites deployed in empty fields were able to calibrate themselves, resolving their positions on the order of meters. An additional mobile rover was necessary to move around the beacon field and refine the positioning numbers to lock the locations down to the centimeter [15]. These proofs-of-concept demonstrate a critical capability for precision surface navigation, such as for science experiments and local autonomous rovers. However, they are also subject to certain downsides that prevent it from being a perfect solution. The signal range is limited to a few hundred meters, and thus would not be practical for surface missions of wider scope. Furthermore, the hardware itself is quite sensitive, easily susceptible to interference, noise, aliasing, and multipath biases [16]. Even high power systems will still be limited to line-of-sight (LOS) conditions [17]. Nonetheless, the capabilities exhibited by these SCPA models should prove to be extremely valuable for smallscale, local operations.

\section{Low-frequency Beyond Line of Sight Approaches}

Low frequency (LF) and very low frequency (VLF) spectrum can provide an alternative to UHF and S-band (Super High Frequency) for usage in beacons applications. Offshoot technologies from the previously mentioned Loran- $\mathrm{C}$ and $\mathrm{ADF} / \mathrm{NDB}$ systems are viable applications for long-term, wide-range lunar surface operations. The primary drawback 
of the higher ranges in the radio frequency spectrum is their LOS limitation. The key advantage of implementing lower frequencies is the ability to operate in situations outside of visual range, or non-line-of-sight (NLOS). Since frequencies on the order of several kilohertz have wavelengths long enough to maintain positive contact between visually disconnected beacons, the reception range subsequently becomes hundreds of kilometers, going well past the limits of the lunar horizon.

These signals propagate in the form of ground waves, which uses the electrical conductivity of the lunar surface to force broadcasted LF and VLF signals to follow the curvature of the planet. This is yet another advantage of the low frequency range. Signals in the high frequency (HF) range and higher attenuate far too easily for ground wave propagation to be a feasible mode of NLOS navigation. For such cases, transmitters that rebroadcast signals between NLOS assets would be necessary for long-range surface tasks, and this increases the number of physical beacons on the surface, increasing lunar descent payload masses as well as required power consumption, and so on. Using lower frequencies, on the other hand, decreases the total mass, power, and volume constraints. While a single LF beacon is much larger and heavier and consumes more power than a single HF beacon, the longer range of LF signals require far fewer total beacons, allowing the mass and power required for a given coverage radius to diminish logarithmically with frequency.

However, there are trade-offs with LF/VLF systems, namely in the form of bandwidth limitations. Due to the lower range of the LF bands compared to the higher frequencies, there is only so much useful data that can be broadcasted. Consequently, the use cases of these lower ranges is limited to basic voice broadcasts as well as data inherent in the signal (such as phased signals, similar to VORs). Thus, these lower frequencies, while suitable for wide-range navigation, should be suspended in favor of high frequencies when fine-tuning to exact locations to take advantage of higher bandwidth local architecture.

\section{Multi-spacecraft Autonomous Positioning System}

With the growth and spread of communication networks out into space to support an expanded presence, there is an opportunity to use these signals as an observable as part of a navigation. The Multi-spacecraft Autonomous Positioning System (MAPS) [18] incorporates with this distributed communication architecture to provide a position estimation capability within the individual spacecraft and nodes that operate within this system. This system works through the embedding of time and state information in a standardized format (including frame and uncertainty information) within every packet shared across the network. Across reception of a packet, a node is thus able to form a one- (or two-way if operating In that mode) ranging and range-rate observation from a source that is broadcasting out it local position. Integration of this information can allow for each assets to perform relative ranging to each other and improve its onboard state knowledge.
Initial studies of this concept focused on simulation results and initial hardware-in-the-loop testing. In the summer of 2018, software implementing this architecture was uploaded to the SCaN SDR Testbed onboard ISS for a series of orbital demonstrations on flight hardware [19]. In this experiment, ranging data was sent via direct to ground, payload telemetry, and through TDRS to be timestamped and it quality assessed for navigation capabilities. The key drivers identified from this study were system synchronization, network latency, and hardware latency. For this system, understanding onboard clock stability and the latencies between packet formation and radio frequency generation drive the performance.

While this system is envisioned as a larger solar system-wide embedded network capability, it is also useful to focus on how well it can integrate into a particularly sub-network. One application of this is to support of navigation in the lunar regime. An example of this architecture is given in Figure 3. In this image, the various trunks between assets are identified, with communication and navigation between spacecraft, ground rovers, and fixed ground stations. This infrastructure mimics the build-out of lunar explorations and can be embedded within these elements to provide distributed capability. This application could support and allow for autonomous operations in the lunar regime, independent of Earth and be a first step towards a much larger network.

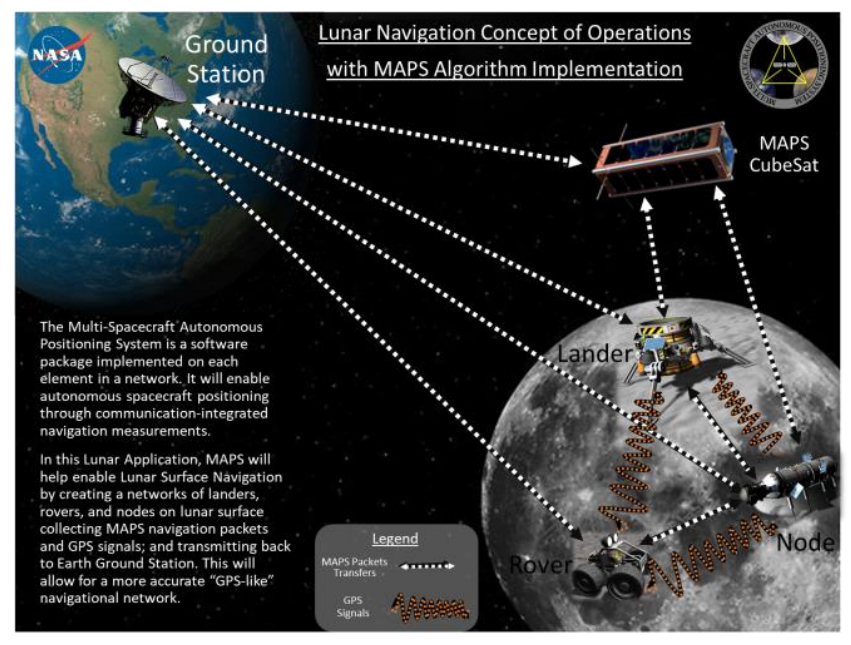

Figure 3: MAPS Lunar Architecture 


\section{LUNAR NODE - 1}

In order to support this architecture, the Lunar Node - 1 (LN1) payload was developed. Its primary objective is to implement the first node in a larger MAPS network, demonstrating the adaptation of the software algorithms to a small, integratable platform. An additional purpose of the payload is to exercise ground links throughout the mission and use the navigation observations to support mission operations and provide insight into a host vehicle's navigation state. $\mathrm{LN}-1$ is being developed to catch a ride as hosted payload on a commercial lunar lander. The lander provides a host interface, including power and commanding and transports the payload to the lunar surface. The payload takes advantage of modern cubesat technology to create a small footprint, lower power platform to allow for demonstration of the MAPS technology.

\section{Concept of Operations}

For this application, the payload will sync its internal time and state to that of the lander from its best estimate. All commanding and health and status will be relayed through primary payload telemetry. The payload includes an independent high stability oscillator and S-band radio to support communications back to Earth. Over the course of the trans-lunar cruise and from the lunar surface, the payload will broadcast out its state and timing information back to Earth for several observation passes via ground networks such as the Deep Space Network. Upon reception of this data, high accuracy packet reception timestamps will be used (along with atmospheric data for induced delays) to assess a ranging observation. This data will be captured across multiple passes to compute a navigation state of the payload over the mission. In addition to demonstrating the MAPS payload, the radio will also be used in standard tone-based non-coherent ranging and Doppler tracking to provide an alternate approach for navigation performance. Figure 4 provides an overview of this operational interface and concept.

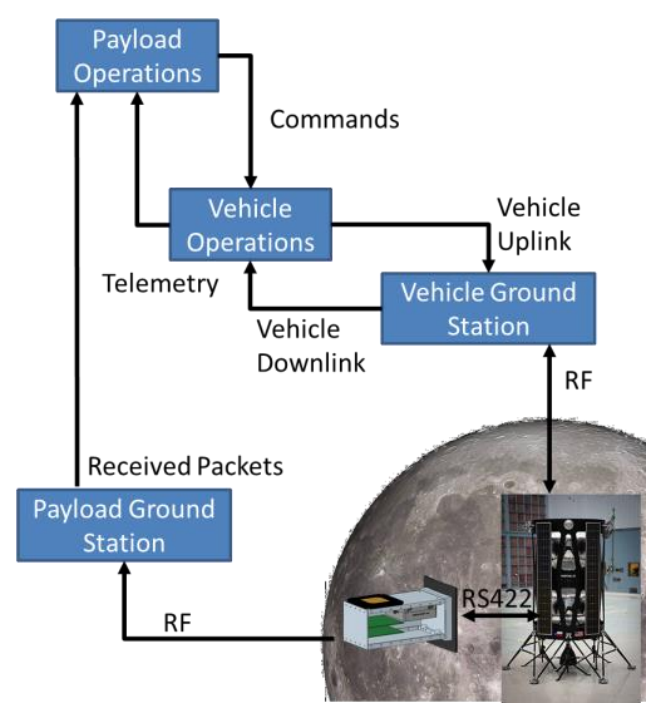

Figure 4: Concept of Operations
Overview of Payload

A model of the payload is given in Figure 5 below. In this drawing, the compact size of the spacecraft can be identified. In terms of dimensions, the primary structure is a little over a $1 \mathrm{U}$ volume. The dominating factor of the design is the large top surface, the spacecraft's radiator. To provide a clean interface with the host vehicle, $\mathrm{LN}-1$ has designed a radiator to allow for heat dumping during operation. This is needed due to the hot environment on the lunar surface, combined with the heat generated by the power draw of the radio while transmitting. While this payload is not being designed to survive the lunar night, it provides a platform that could be integrated into a host vehicle and with adequate power generation/storage be able to offer long term operation.

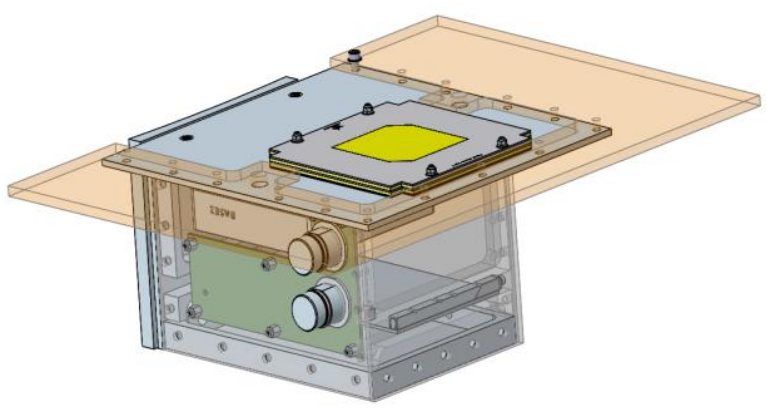

Figure 5: Lunar Node -1 Beacon Payload

\section{Demonstration Capability}

In order to show the performance of this system, the ranging observations will be used in a ground orbit determination filter to calculate a navigation fix for comparison to external measurements. The demonstrated capability is a function of observation time and measurement error. To quantify this ranging capability, a simulation was created using FreeFlyer, a mission analysis software, and external Python scripting. Within FreeFlyer, a stationary lander was at an assumed location on the Moon's Oceanus Parcellum with a July 2021 epoch. Simulated Deep Space Network (DSN) range measurements, with noise, were generated at 60 second time steps.

These measurements were processed using a nonlinear least squares batch filter script. The lander's position was solved for in a lunar-centered-lunar-fixed (LCLF) frame. The simulation used a Gauss-Newton iteration (Eq. 2) to estimate the lander's location by minimizing the sum of the residuals squared (Eq 3).

$$
\begin{gathered}
r_{x, y, z}^{n+1}=r_{x, y, z}^{n}-\left(\boldsymbol{J}^{T} \boldsymbol{J}\right)^{-1} \boldsymbol{J}^{T} \boldsymbol{Y}\left(r_{x, y, z}^{n}\right) \\
r s s=\sum_{i=1}^{m} y_{i}^{2}\left(x, y, x, t_{i}\right)
\end{gathered}
$$

In this equation, $r$ represents the lander's LCLF position, $Y$ represents the range estimate, and $J$ is the Jacobian matrix. 
A 1000 case Monte Carlo was run for each of the combinations of observation time intervals and noise levels. Observation time intervals vary in length between 1 and 7 hours. Gaussian measurement noise levels vary from $0.01 \mathrm{~km}$ to $3 \mathrm{~km}$ of range error. Figure 6 shows the results of this analysis.

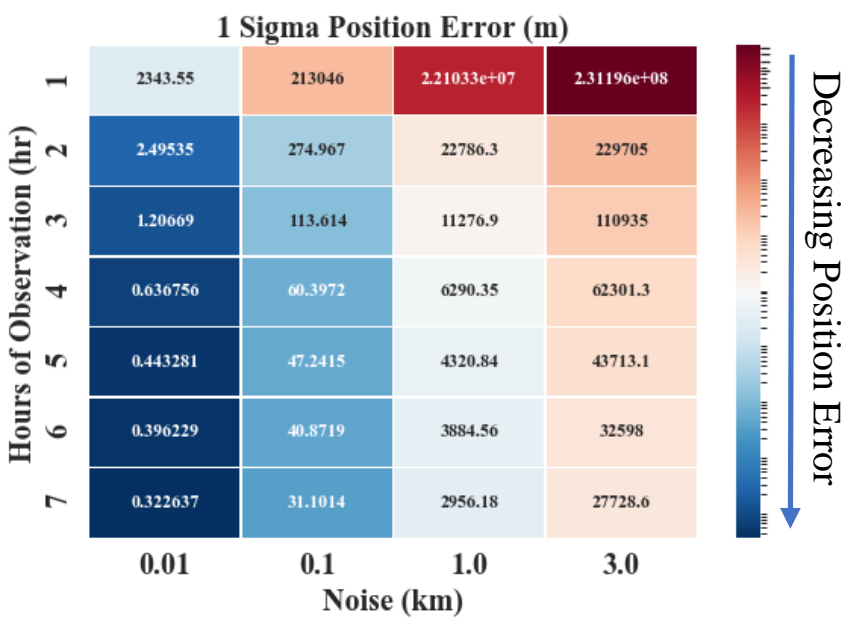

Figure 6: Position Accuracy, in meters, as a Function of Noise and Observation Time

The desired position accuracy for LN-1 is less than $100 \mathrm{~m}$. Figure 6 shows that this position knowledge can easily be obtained if noise is less than or equal to $0.01 \mathrm{~km}$ given an adequate duration of continuous duration. This analysis assumed measurements at $1 \mathrm{~Hz}$ intervals over a fixed duration. The analysis assumed continual DSN availability and did not contain any drop-outs, which may occur in an operational setting. The $0.1 \mathrm{~km}$ noise results show that longer observation times will be required, but it is still possible to achieve position accuracy. Noise levels up to $1 \mathrm{~km}$ ranging do not resolve within $100 \mathrm{~m}$ on DSN observations alone in a reasonable time frame.

It is important to note that DSN observations will be limited on availability as measurements can only be acquired for short durations each day. Understanding how this constraint would affect this demonstration is a necessary metric for mission planning. To show this, Figure 7 displays the resulting 1-sigma error over time if only a limited amount of observations could be taken daily.

Figure 7 shows the results state prediction for $0.1 \mathrm{~km}$ of noise on the observations. This noise value was chosen to analyze because, as shown in Figure 6, longer observation times are required to reach state accuracy. Figure 7 shows that two or three hours of observation can be made each day, it would at most 2 days to obtain the required state knowledge. However, if only one hour of observation was taken each day, it would take up to four days to obtain the needed state knowledge.

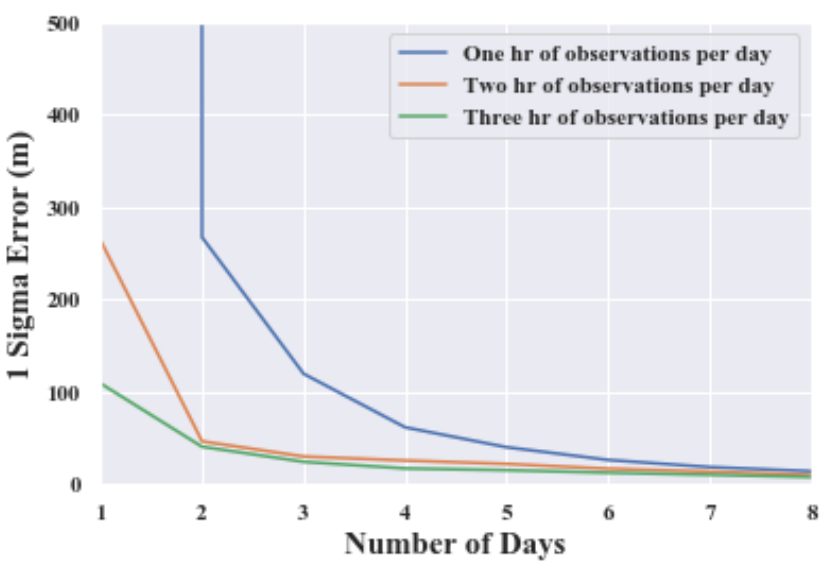

Figure 7: 1-Sigma Position Knowledge over Time for 0.1 km of Measurement Error

\section{Applications to Future Missions}

The previous sections describe how a beacon can be placed on the lunar surface and its capability in order to calibrate its initial state estimate. This next section provides an overview of a variety of use cases of beacons within the proposed lunar architecture. These applications all build on the functionality implemented by the LN-1 beacon hardware. These subsections intend to provide insight into potential applications and notional first order analysis for expected performance. The missions described herein focus on the initial roll-up of the beacon network capability, with one beacon providing operational navigation support to a variety of missions.

Four primary operational modes are described in the following sections. These ties to the mission phases being proposed as part of the return to the Moon and lunar missions being developed by commercial partners to meet NASA goals and capabilities. The individual missions also present use cases where the LN-1 hardware could be bootstrapped into upcoming missions to build out the navigation networks. The missions under discussion are: orbit determination from a ground beacon, ground navigation from an orbiting beacon, supporting high precision landing, and navigation observations during ascent from a lunar platform. Additionally, many of these applications are planetary-body agnostics, and are directly applicable to future mission scenarios, such as a human Mars and large cargo missions.

\section{Orbit Determination-Ground Beacon}

One of the first functions that a calibrated ground beacon can provide is to support local orbit determination about a planetary body. This case directly resembles standard ground-based orbit determination methods, in which as a spacecraft passes overhead, range and range-rate data is collected. This data is fed into a nonlinear filter to provide a best estimate of the spacecraft's positon. For Earth-based applications this data is collected and processed on the surface with a resulting vehicle state captured for future estimation and mission planning. In this scenario, the 
spacecraft's onboard processing would be the primary recipient of ranging data to provide on onboard solution autonomously. This would allow for an orbiting asset to run an onboard filter and process ranging and range-rate observations to updated onboard state knowledge. With the ground beacon having a fixed position, its ability to provide ranging capability is tied to its onboard clock stability, though external methods (such as star trackers, atomic clocks, or ranging back to earth) can help to provide external disciplining of the system.

\section{Orbit Determination -Orbital Beacon}

Another scenario under consideration is the integration of a navigation beacon onto an orbiting asset. This assets could be a local orbiting relay in a low lunar orbit or further out in a NRHO-type orbit about a Lagrange point. This is the reverse application to that just described. For this scenario, the beacon syncs its time and state to that provided by the spacecraft. This can either be provided by a high accuracy Earth-observed solution or be onboard methods such as optical navigation, $\mathrm{x}$-ray navigation, or the use of low power GNSS receivers with software capable of operation in lunar orbit [20].

Similarly to the previous case, the beacon provides one-way ranging information between the spacecraft and the receiving vehicle on the lunar surface by transmitting out onboard time and state. To assess this scenario, a spacecraft was assumed to be in a $200 \mathrm{KM}$ altitude polar orbit about the moon. This spacecraft's onboard knowledge is assumed to be maintained by an onboard GNSS Receiver [21]. These results show the capability of a ground assets estimating its position over a maximum of 11 passes, with each pass being 10 minute passes every two hours for a south pole-landed vehicle.

Figure 8 provides a contour of estimated ground state as a function of ranging measurement error. In this analysis a nonlinear least squares filter was implemented to process the ranging observations and provide a best estimate of the landing site. The $\mathrm{x}$-axis captures the error in the ranging measurement on a log-10 scale. The y-axis identifies the number of passes of observations used in the integrated state estimate. The results from this plot show that the accuracy is limited by the noise of the filter, and that $100 \mathrm{~m}$ positioning accuracy is easily achieved for $>3$ passes with measurement errors $<100 \mathrm{~m}$. As the errors increase, more time is needed to get an as accurate solution. The caveat to this analysis is the assumption of perfect knowledge of the beacon's state.

Figure 9 shows the effect of beacon knowledge error on the ground state determination. For this scenario, external analysis captured the onboard state accuracy of the spacecraft across the orbit. In this case, in addition to providing a range observations the beacon also provided/broadcast its own onboard estimated state. In this case, the spacecraft's state is assumed to be of better accuracy than the ground asset. This chart has similar $\mathrm{x}$ - and $\mathrm{y}$-axis to the previous chart, but it is important to note that the contours show the log-10 of the position error. In this scenario, the onboard state knowledge directly limits the ability of the ground assets to measure its position. Another key takeaway in this analysis is that given a value of measurement error, the onboard state knowledge limits the accuracy of the system, requiring many more passes to get the same accuracy.

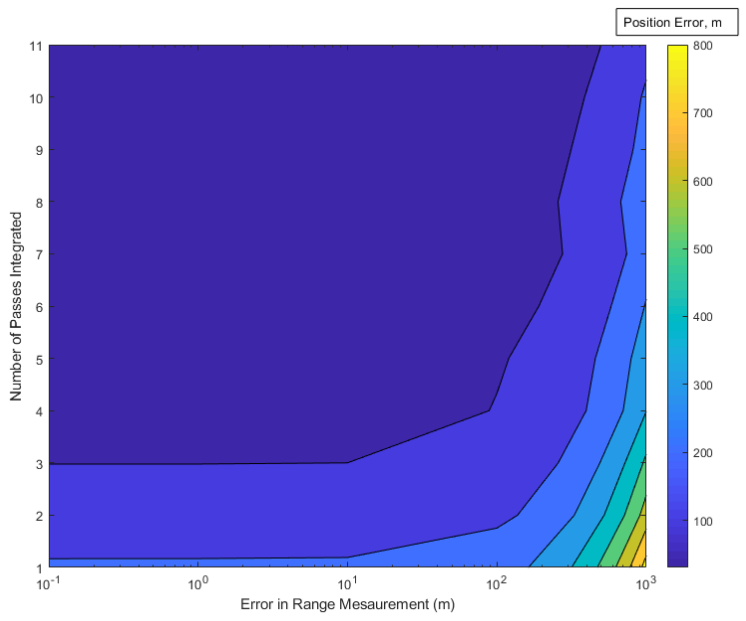

Figure 8: Ground Determination Accuracy with Orbital with Perfect State Knowledge

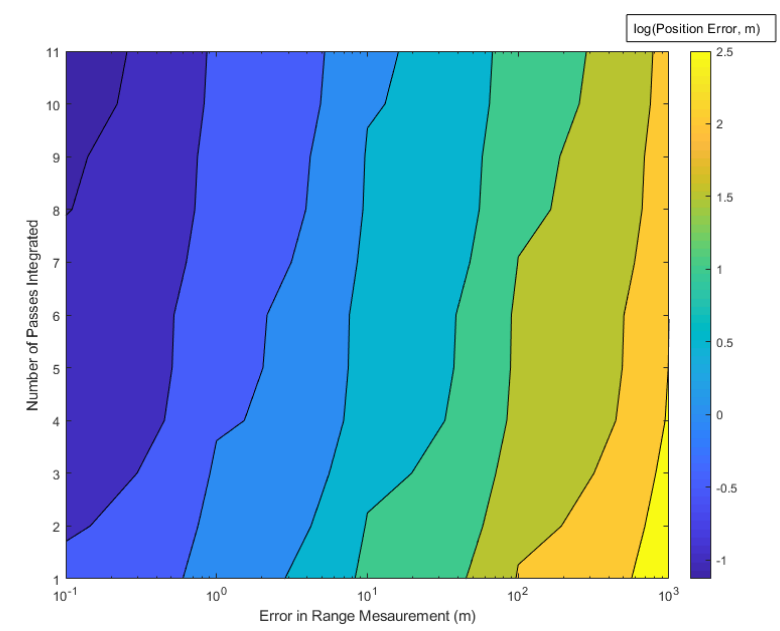

\section{Figure 9: Ground Determination Accuracy from an Orbiting Beacon with Onboard State Uncertainty}

In this scenario, the knowledge errors of the onboard spacecraft effectively bias the observation away from a zero mean observation. This helps to explain the difference in behavior between the two scenarios. Additionally, this filter used in this case assumed perfect knowledge of the orbiting asset. Filter maturation and more sophisticated approaches can help to account for this accuracy and provide improved performance.

\section{Lunar Ascent - Ground Beacon}

In terms of supporting lunar operations, an alternate approach is to operate similar to terrestrial airports that provide local navigation information for ascent and takeoff. This 
application assumes a stacked lander and ascent vehicle configuration starting from the lunar surface. In this scenario, similar to the LN-1 operational plans, the beacon is placed on the descent element and is calibrated upon landing on the lunar surface. Using landing accuracy validation data, the beacon is provided with a best estimate of its location on the lunar surface and can sync to an external time source. The primary application of this beacon is to provide support for its return element during ascent flight form the lunar surface and for future descent missions to the same area (discussed in the next section).

In this analysis scenario, the beacon is assumed to have essentially perfect knowledge of its state onboard the lunar surface and provides a ranging measurement to the ascending vehicle. For this trajectory, it is assumed that once the elevation angle between the ascent vehicle and the lower horizon (relative to the vertical) is over 75 degrees, the line of sight is lost and the measurement is lost. Figure 10 shows the dispersions of the inertial $\mathrm{x}$-position errors during the ascent trajectory. The initial position knowledge was assumed to be 100 meters. The trajectory flown is representative of a human lunar ascent mission. Without any external observations, the errors continue to grow. The first 100 seconds represent a fairly vertical ascent to gain altitude followed by a gravity-turn like maneuver to enter into a lunar orbit, hence the growing large growth in this axis after 200 seconds.

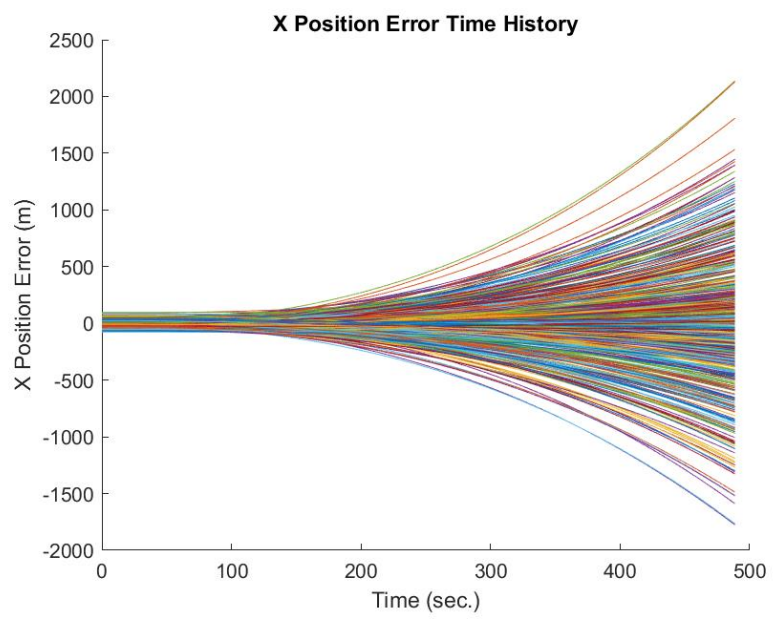

Figure 10: Baseline Inertial only performance

Figure 11, though, includes the integration of an onboard beacon. Again, the limitations on line of sight are implemented to provide insight into actual capability. The vehicle used a 6 state Extended Kalman Filter, composed of positon and velocity errors states to integrate the ranging measurements. In this plots, it can be seen how the beacon helped to reduce errors early in flight to a much tighter level during the first 100 seconds of flight. As the vehicle turned downrange, the effectiveness of the measurement in this axis is reduced and the inertial errors grow similarly to the previous scenario.

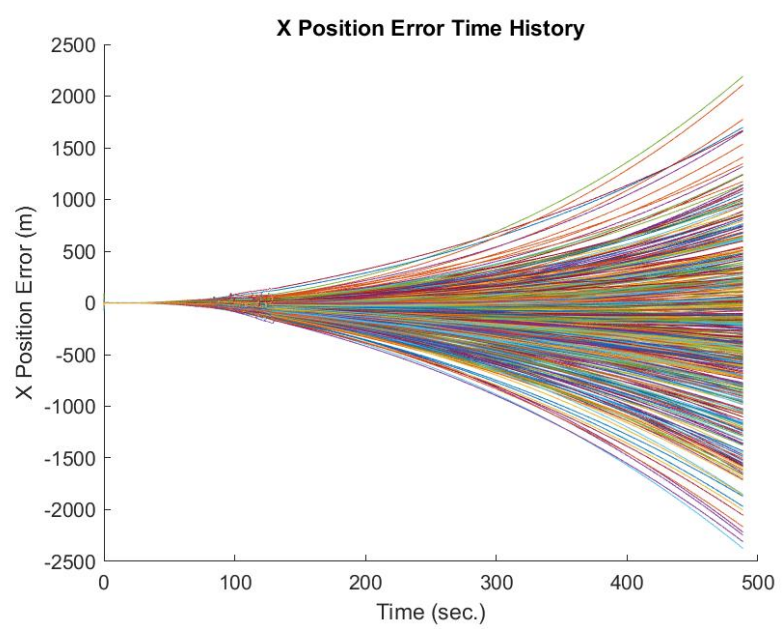

Figure 11: Beacon Implemented at Launch Site

This performance is due to the limited observability of the ranging observation in this filter implementation. The range (and range-rate) provide insight into the position and velocity errors, though primary along the ranging vector from the spacecraft to the ground beacon. As the spacecraft travels father away, the primary observation inertial axis will change, providing only secondary insight to each axis (via the sensitivity matrix definition, $\mathrm{H}$, providing the change in range due to changes in each axis). Additionally, since the range does not directly measure a specific axis, there are multiple solutions (positions) that can provide the same range. For this, proper tuning of the filter is necessary to achieve good performance. Another limitation of this filter is its lack of attitude error states. That state could provide a second order interaction between attitude errors and position/velocity errors. This use case shows promise, and continued maturation of the filter, further tuning, and integration of additional beacons can help to improve the capability.

\section{Precision Landing - Ground Beacon}

The last scenario of discussion is also directly related to terrestrial aircraft navigation support. In this application of navigation beacons, a node is placed at or near a desired landing site and is used to provide ranging information to vehicle during its descent and landing operations. Currently, for human landing systems, high precision landing requirements are being levied on vehicle designs (such as the VIPER lander [22]) to demonstrate technology and ensure repeatability/capability for future missions. In order to achieve this, landing vehicles require an extensive suite of navigation sensors to provide navigation observations and maintain an accurate state. Two of the primary technologies are Terrain Relative Navigation [23] and laser altimetry/velocimetry. An example sensor of this type is the Navigation Doppler LIDAR [24], which provides 3-axis range and range-rate information relative to the planetary surface. These, combined with a navigation grade IMU and an accurate initial state estimate prior to powered descent, have been shown to meet high accuracy landing requirements [25]. Figure 12 shows a baseline scenario for a notional human lunar descent trajectory. At beginning of flight, the 
vehicle is flying purely inertial due to the high thrust and limited observability of the onboard optical sensors. Past 400 seconds, the TRN system comes online providing an absolute position observation greatly reducing errors. Then at 500 seconds the altimeter/velocimeter enters its operational range further reducing onboard position and velocity errors. With this sensor suite the vehicle is able to land extremely precisely and accurately.

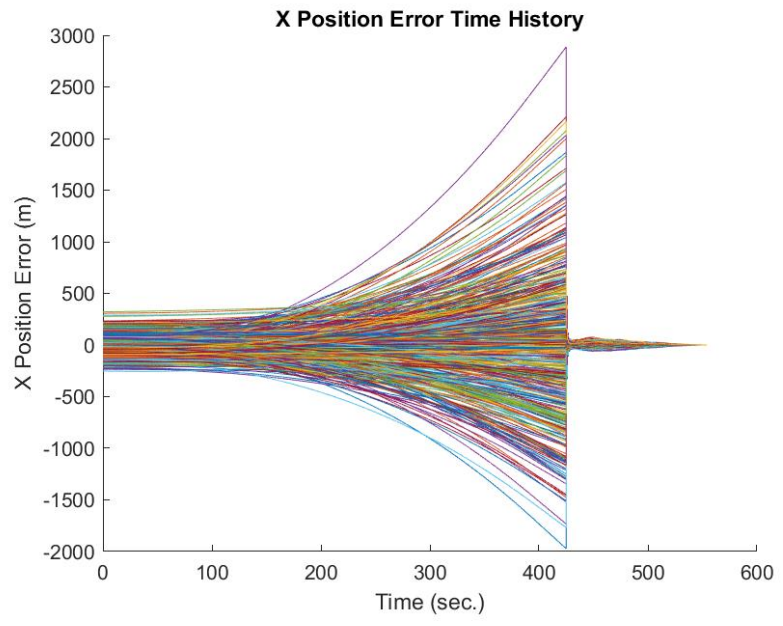

Figure 12: Descent Baseline

An augmenting capability is the inclusion of the navigation beacon into the architecture. The benefits of the beacon is that it can provide landing site relative information and with enough power reach higher altitudes than the TRN camera can, whose functionality is limited to its onboard maps (and their resolution). To assess, a detailed open-loop 6 degree of freedom trajectory was used with high fidelity sensor models and 9 state Extended Kalman Filter (position, velocity, and accelerometer bias errors). Again, in this application, the attitude estimation was decoupled from the translational filtering. Future development is poised at integrating these into one filter. One rationale for this is the extended use of the attitude filter for cruise operations when no translational observations are available.

In this case, shown in Figure 13, the beacon is able to provide navigation knowledge much earlier in the descent trajectory, providing observations at 200 seconds from descent. Note that for this trajectory design, the vehicle comes in at a very high altitude over the landing site and quickly performs a braking burn and begins descent. In this case, the errors are bounded early on, and maintained through the TRN observations. Similarly, upon the operation of the velocimeter/altimeter, the navigation errors further tighten up. This is due to the multi-axis range and range-rate observations with very high accuracy (order of magnitude other that assumed for the ranging observation). In this case, as well, high precision landing is enabled. With this additional capability, it is possible to look at the overall sensor architecture and assess trades between.

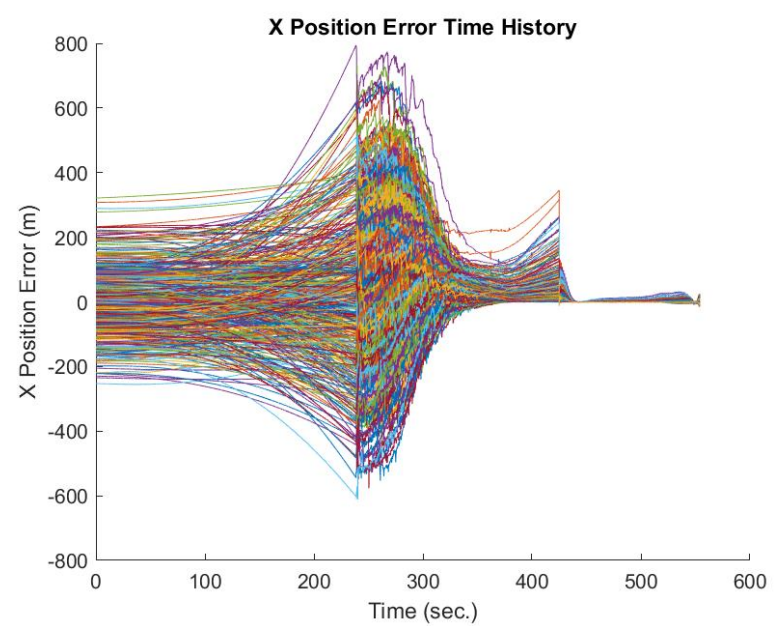

Figure 13: Descent with Beacon

The results of one such study are given in Figure 14 below. In this analysis, the beacon and TRN are still enabled, but the altimeter/velocimeter is removed. As seen, this primarily affects the end of flight. When the position errors begin to increase again. One reason for this behavior late in flight is due to the geometry of the observation of the measurement itself. As the vehicle comes in over the beacon at the landing site, it has very good insight in the vertical axis (due to the primary vertical descent at this stage). The observation of range doesn't provide as much insight into the lateral directions and this is seen in the plot, in the inertial $\mathrm{x}$-axis.

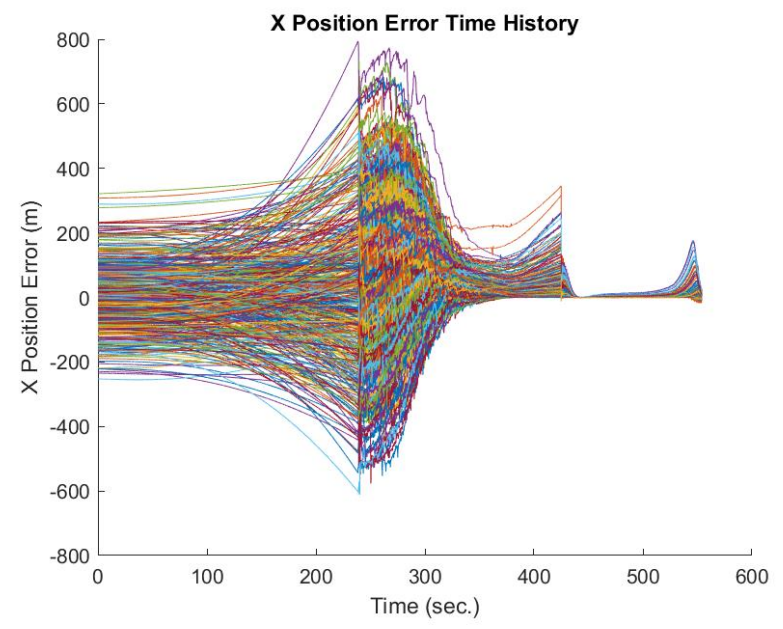

Figure 14: Descent with Beacon but without NDL

\section{NETWORK AND CAPABILITY EXPANSION}

\section{Surface Navigation - Low Frequency non-LOS}

In certain conditions during surface operations, circumstances may arise where crew members are not within line of sight of each other. Astronauts who conduct a sortie to the other side of a hill or who decide to explore the inside of a deep lava tube have no guarantee of a MAPS satellite flying overhead, leading to little chance of maintaining contact with company assets during such scouting missions, and with a 
great enough distance, the standard $\mathrm{S}$-band radios will be ineffective. The solution is to set up a low frequency beacon infrastructure to ensure positive contact during NLOS situations. A network of two beacons can cover a radius of hundreds of kilometers, and a system of less than ten is enough to encompass a complete lunar hemisphere, such as the entire near side of the Moon. In comparison, a high frequency beacon would cover a radius of less than 3 kilometers, and would require an infrastructure of well over a thousand to cover one face.

LF beacons would complement higher frequency ones by adequately by providing a "lower-resolution," yet efficient, option for extensive surface operations, such as for crewed rovers, where S-band beacons would not only be excessive from an accuracy perspective, but the range would also not allow for an adequate travel radius. Systems like LN-1, however, would certainly come into play during ventures that require higher precision routing, such as for science experiments, as well as for congested areas such as a base camp, where the higher bandwidth would provide for more channel flexibility and availability. While they are more coarse than LN-1 and similar systems, LF networks tend to be more robust and reliable. Accordingly, the implementation of low frequency groundwork would efficiently balance the use cases of LN-1 in favor of global navigational capabilities, while allowing $\mathrm{S}$-band infrastructure to take over for smaller scale direction-finding.

Additionally, this payload can be expanded to operate as a communication nodes in a larger networking, for example, acting as a node in a Delay Tolerant Network (DTN). This capability is intrinsic to the high level MAPS architecture and provides a venue and opportunity for the inclusion of navigation information between assets in the network. With the inclusion of non-LOS functionality, the hardware could both support low bandwidth long range operations and transition to higher bandwidth, higher frequency operations as the elements approach each other, providing both robustness and flexibility.

\section{SUMMARY}

This research provides an overview and approach to the first integration of navigation beacons into extraterrestrial applications. The Lunar Node -1 payload provides a hardware testbed for lunar demonstration of the technologies involved, focusing on small-size cubesat-class components to operate within a lunar environment. With the inclusion of high accuracy timing components, the ranging capability of this system is increased. Further ground testing will expand the functionality of the hardware platform, taking advantage of the existing interfaces to work with and develop a range of beacon implementation functionalities. A primary future interest is also in developing analog methods, such as VOR, into a small package, that provide both ranging and bearing information relative to a target. Similarly, improvements to the onboard navigation algorithms in their processing of the flight data can help to further improve expected capability. The analysis shows the capability improvements possible with a first step of beacon deployment. Future studies will continue to refine these results, delving deeper into integration with 6DOF simulations, beacon payload, filter integration, and approach trajectory sensitivities.

\section{ACKNOWLEDGEMENTS}

The team would like to acknowledge the support and funding of the LN-1 payload as part of the NASA Provided Lunar Payload program. The development of that payload is large efforts amongst the team and credit is due to the hard work of Randall Montgomery, Garrick Merrill, Brian Gibson, Thomas Brooks, Jarret Bone, Brian Sturges and Patrick Campbell. We would also thank our program support, Anthony Guillory, Steve Elrod, Howard Soohoo, Frank Moreno, and Ryan Stephan. Lastly, we would like to thank our EV42 management for being ever supportive of this technology development and research, particularly Heather Koehler.

\section{REFERENCES}

[1] K. Natarajan, "Use of Loran-C for General Aviation Aircraft Navigation," in NASA Langley Research Center Joint Univ. Program for Air Transportation Res., Cambridge, Massachusetts, USA, Mar. 1, 1981.

[2] Federal Aviation Administration. (1988, Aug. 24). AC 20121A, Airworthiness Approval of Loran-C Navigation Systems for use in the U.S. National \& Airspace System (NAS) and Alaska (Cancelled). [Online]. Available: FAA Home: Regulations \& Policies: Advisory Circulars

[3] J. G. Lucas, et al., "VHF Omni-range Navigation System Antenna," U.S. Patent 4451830, May 29, 1984.

[4] Civil Aviation Safety Authority. (2005). Operational Notes on Non-Directional Beacons (NDB) and Associated Automatic Direction Finding (ADF). [Online]. Available: FAA Home: Regulations \& Policies: Advisory Circulars

[5] A. Pue and M. Vaujin. (2019). Course 557-1906: Inertial Systems, Kalman Filtering, and GPS/INS Integration [PowerPoint slides].

[6] J. L. Goodman, "GPS Lessons Learned from the International Space Station, Space Shuttle, and X-38," NASA Johnson Space Center, Houston, TX, USA, NASA/CR-2005-213693, Nov. 1, 2005. 
[7] Federal Aviation Administration. (2015, Apr. 21). AC 90-108, Use of suitable Area navigation (RNAV) system on Conventional Routes and Procedures - Change 1. [Online]. Available: FAA Home: Regulations \& Policies: Advisory Circulars

[8] Strohmeier, et al., "Realities and Challenges of NextGen Air Traffic Management: The Case of ADS-B," IEEE Communications Magazine, vol. 52, no. 6, pp. 111-118, May 19, 2014.

[9] Federal Aviation Administration. (2015, Dec. 7). AC 20165B, Airworthiness Approval of Automatic Dependent Surveillance - Broadcast OUT Systems. [Online]. Available: FAA Home: Regulations \& Policies: Advisory Circulars

[10] Air Land Sea Application Center. (2000, June 30). TADIL J: Introduction to Tactical Digital Information Link J and Quick Reference Guide. [Online]. Available: Defense Technical Information Center: Documents

[11] H Qin, et al., “A JTIDS/INS/DGPS navigation system with pseudorange different information transmitted over Link-16: design and implementation," The Journal of Global Navigation Satellite Systems, vol. 17 pp. 391-402, Sep. 18, 2012.

[12] W. S. Widnall and J. F. Kelley, "JTIDS Relative Navigation With Measurement Sharing: Design and Performance," IEEE Transactions on Aerospace and Electronic Systems, vol. AES-22, no. 2, pp. 146-154, Mar. 1986.

[13] C. D. Edwards, et al., "The Electra Proximity Link Payload for Mars Relay Telecommunications and Navigation," in $54^{\text {th }}$ Int. Astronautical Congr., Bremen, Germany, Sep. 29, 2003.

[14] E. G. Lightsey, et al., "Real-Time Navigation for Mars Missions Using the Mars Network," Journal of Spacecraft and Rockets, vol. 45, no. 3, pp. 519-533, May-June 2008.

[15] J. Stone, et al., "GPS Pseudolite Transceivers and their Applications" in ION Nat. Tech. Meeting 99, San Diego, California, USA, Jan. 25-27, 1999.

[16] J. Wang, "Pseudolite Applications in Positioning and Navigation: Progress and Problems," Journal of Global Positioning Systems (2002), vol. 1, no. 1, pp. 48-56, July 18, 2002.

[17] E. LeMaster and S. M. Rock, "A Local-Area GPS Pseudolite-Based Mars Navigation System," in IEEE $10^{\text {th }}$ Int. Conf. on Advanced Robotics, Budapest, Hungary, Aug. 2001.

[18] E. Anzalone, C Becker, D Crump, and D. Heater, "Multispaceraft Autonomous Positioning System: LEO Demo Development," in AIAA/USU Small Satellite Conference, Logan, UT, Aug. 2015.
[19] E. Anzalone, C. Becker, and H. Sims, "Initial Results of the Software-driven Navigation for Station Experiment" in AIAA SPACE and Astronautics Forum and Exhibition, Orlando, FL, Sept. 2018.1

[20] L. Winternitz et al., "GPS-Based Autonomous Navigation Study for the Lunar Gateway", AAS 19-096, in Proceedings of the AAS 42nd Annual Guidance and Control Conference, February 2019.

[21] E. Anzalone, J. Getchius, J. Legget, et al, "Lunar Navigation Beacon Network using Global Navigation Satellite System Receivers", in International Astronautical Congress, Washington, D.C., October 2019.

[22] J. Orphee, M. Hannan, et al, "Guidance, Navigation, and Control for the NASA Lunar Pallet Lander" in Proceedings of the AAS 42nd Annual Guidance and Control Conference, February 2019. AAS 19-033.

[23] Johnson, Andrew E., and James F. Montgomery. "Overview of terrain relative navigation approaches for precise lunar landing." In 2008 IEEE Aerospace Conference, pp. 1-10. IEEE, 2008.

[24] Pierrottet, Diego, Farzin Amzajerdian, Larry Petway, Bruce Barnes, and George Lockard. "Flight test performance of a high precision navigation Doppler lidar." In Laser Radar Technology and Applications XIV, vol. 7323, p. 732311. International Society for Optics and Photonics, 2009.

[25] E. Anzalone, E. Braden, et al. "Guidance and Navigation Design Trades for the Lunar Pallet Lander", in Proceedings of the AAS 42nd Annual Guidance and Control Conference, February 2019, AAS 19-09

\section{BIOGRAPHY}

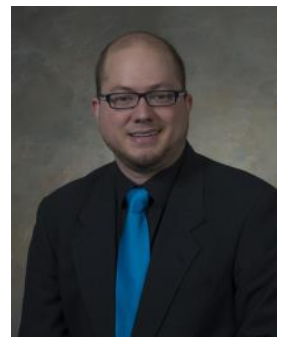

Evan Anzalone received a B.S. in Physics from Louisiana State University in 2006. He completed his Master of Science Degree at Georgia Institute of Technology in 2008, and PhD in Aerospace Engineering in 2013, with his thesis, "Agent and model-based simulation framework for deep space navigation analysis and design." He has been with NASA/MSFC for 9 years. He currently serves as a Navigation SME for the EV42 Guidance, Navigation, and Mission Analysis branch. He co-leads the SLS Navigation Team and supports system architecture, hardware review and specifications, performance analysis, and fault detection and isolation analysis. He is also the inventor and developer of the Multi-spacecraft Autonomous Positioning System, and PI of the Lunar Node -1 payload, which will demonstrate lunar navigation beacon hardware in 2021. In addition to SLS, Evan is also the Navigation lead for a Martian 


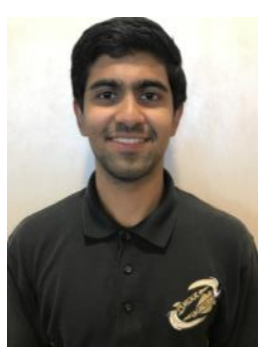

Anand Iyer received a B.S. in aerospace engineering from Purdue University in 2018. He works as a Guidance, Navigation, and Control Engineer supporting lunar landers, Martian ascent vehicles, and the Space Launch System at NASA Marshall Space Flight Center. He is also a pilot and flight instructor.

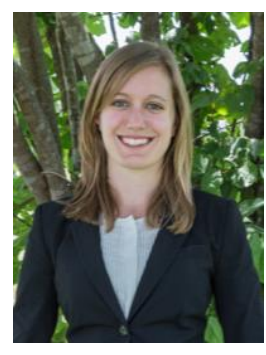

Tamara Statham is currently working as a Guidance, Navigation, and Control Engineer $(G N \& C)$ with NASA Marshall Space Flight Center. In this role, she supports $G N \& C$ analyses for NASA's Space Launch System (SLS) and Artemis programs. Prior to her position with NASA, she worked with Jacobs Technology supporting SLS trajectory development, and with the U.S. Army Space and Missile Defense Command supporting small satellite technology development for communication and imaging relays.

Tamara has received the NASA Space Flight Awareness Trailblazer Award and the Army's Achievement Medal for Civilian Service in her past positions. She holds a B.S. in mechanical engineering from the University of Alabama in Huntsville and an M.S. in aerospace engineering from the University of Colorado at Boulder with a focus in astrodynamics and satellite navigation. She is an active member of American Institute of Aeronautics and Astronautics (AIAA) where she is currently the Region II Deputy Director for Young Professionals. 